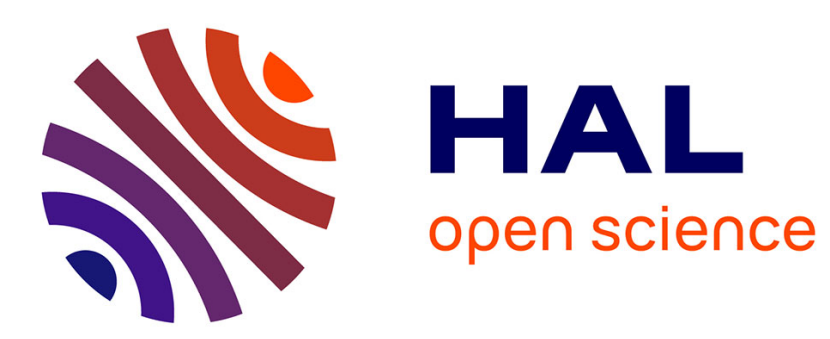

\title{
Robust Control of a Single-Ended Primary-Inductor Converter (SEPIC)
}

Roushan Rezvani, Antoneta Iuliana Bratcu

\section{To cite this version:}

Roushan Rezvani, Antoneta Iuliana Bratcu. Robust Control of a Single-Ended Primary-Inductor Converter (SEPIC). ISEEE 2017 - 5th International Symposium on Electrical and Electronics Engineering, "Dunarea de Jos" University of Galati, ROMANIA, Oct 2017, Galati, Romania. hal-01592360

\section{HAL Id: hal-01592360 \\ https://hal.science/hal-01592360}

Submitted on 30 Sep 2017

HAL is a multi-disciplinary open access archive for the deposit and dissemination of scientific research documents, whether they are published or not. The documents may come from teaching and research institutions in France or abroad, or from public or private research centers.
L'archive ouverte pluridisciplinaire HAL, est destinée au dépôt et à la diffusion de documents scientifiques de niveau recherche, publiés ou non, émanant des établissements d'enseignement et de recherche français ou étrangers, des laboratoires publics ou privés. 


\section{Robust Control of a Single-Ended Primary-Inductor Converter (SEPIC)}

\author{
Roushan Rezvani \\ Linköping University \\ 581 83, Linköping, SWEDEN \\ Email: karre337@student.liu.se
}

\author{
Antoneta Iuliana Bratcu \\ GIPSA-lab, Grenoble INP \\ 38402, Saint-Martin d'Hères Cedex, FRANCE \\ Email: antoneta.bratcu@gipsa-lab.grenoble-inp.fr
}

\begin{abstract}
This paper approaches the topic of robust control for DC-DC power converters. A low-power single-ended primaryinductor converter (SEPIC) has been chosen as a case study, for which the output voltage regulation problem in presence of parameter uncertainties is stated in the $H_{\infty}$ formalism as a disturbance-rejection problem. This approach is assessed by numerical simulations in MATLAB ${ }^{\circledR}$ against a classical integral state-feedback control from the point of view of robustness in stability and in performance, when two parameters - input voltage and load resistance - vary, but are bounded within known ranges. Comparison shows that $H_{\infty}$ control design, provided that it handles more degrees of freedom, can offer more detailed information about robustness ranges, but this advantage has to be weighed against its complexity compared to state-feedback control. Choice of some either classical or robust control solution depends upon requirements and constraints of each application.
\end{abstract}

\section{INTRODUCTION}

Power electronic converters are switching devices used for controlling the electric energy flows within power structures, generally aiming at conditioning the power flows with respect to a certain application. Nowadays context of power and energy applications - in continuous growth and development such as to respond to increasing energy needs - requires ubiquity of power converters as flexible actuators able to ensure some desired performance - stability, very fast response, robustness - under strong constraints.

Power converters may accomplish various basic tasks; in particular, DC-DC converters are fed with DC voltage and they output DC voltage with different value and possibly different polarity than the input voltage. The main control goal in DC-DC converters may vary upon their role, but the general control scope is to regulate/track either the output or the input converter voltage (with respect to the power flow), while meeting a set of imposed performance requirements. The control structure may be more or less complex, as the primary goal may generate subgoals defined when other issues are revealed during modelling (e.g., stability or robustness aspects)

The topic of robust control of DC-DC converters has previously been studied. DC-DC converters are nonlinear systems and a common control approach has been to disregard the nonlinearities and consider a small-signal model valid around an operating point.
It has been shown that the direct voltage control of SEPIC reveals a fourth-order non-minimum-phase system [4]; indirect voltage control based on a two-loop structure with an inductor current inner control loop is a common method to deal with this issue [1]. Linear controllers are usually employed to this end, but their intrinsic robustness may be challenged under parameter variations and lead to unexpected behaviour in the presence of uncertainties; this is why robustness of various control laws is an interesting issue.

Different methods to robustify control structures for DCDC power converters have been proposed, among which one can cite those based on shaping the frequency response. A proportional-integral (PI) controller for a SEPIC is designed by loop shaping in [5], whereas in [6], a robust PID controller for a buck converter is proposed using an $H_{\infty}$ approach. A controller is designed in [7] by use of $H_{\infty}$ control theory, in which the nonlinear characteristic of the system has been modeled as uncertainties and the obtained controller has superior performance when compared with an LQR controller. Robust output voltage control is obtained for a high-gain DCDC converter in [8] by the use of a sliding-mode controller. Sliding-mode control is particularly adapted for power converters, whose dynamics are switching at high frequency ([9], [10]).

This paper investigates the pertinence of an $H_{\infty}$ controller designed by the use of weighting functions as a way to robustify the closed-loop performance of a single-ended primaryinductor converter (SEPIC) in the presence of uncertainties, namely those coming from input voltage variations and those given by load resistance variations.

The control problem is stated as the output voltage regulation and is cast into the $H_{\infty}$ formalism as a disturbancerejection problem. Results of this approach are assesed by numerical simulation against those of a classical linear statefeedback control with integral action.

This paper is organized as follows. Section II, which is dedicated to modelling aspects, is followed by state-feedback control design in Section III and then by $H_{\infty}$ control design in Section IV. Section V discusses some simulation results, whereas Section VI allows to compare the two approaches from the point of view of sensitivity and robustness to parameter variations (input voltage and load resistance). Section VII concludes this paper. 


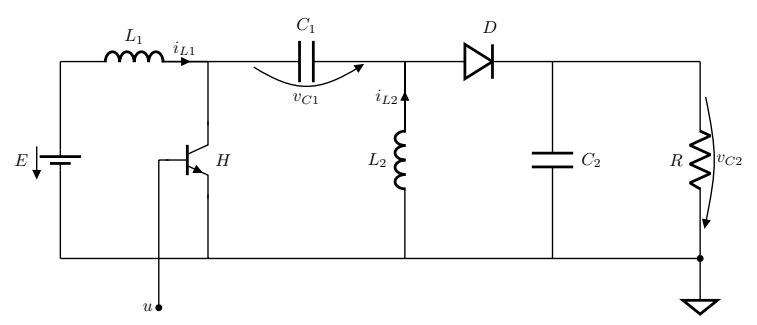

Figure 1. Circuit diagram of the SEPIC converter.

\section{SEPIC MODELLING}

A SEPIC converter is a DC-DC converter driven by the binary switching function $u$, giving it two configurations. It is capable of providing an output voltage $v_{C 2}$ both larger and smaller than the input voltage $E$ [1]. Fig. 1 gives the circuit diagram of a low-power SEPIC converter. The values of the circuit components are the following: input voltage $E=4.5 \mathrm{~V}$ (can vary between 3 and $5.7 \mathrm{~V}$ ), output voltage setpoint $v_{C 2}^{*}=3.3 \mathrm{~V}, L_{1}=L_{2}=4.6 \mu \mathrm{H}$, $C_{1}=10 \mu \mathrm{F}, C_{2}=200 \mu \mathrm{F}$, load resistance $R=1.3 \Omega$ (can vary between 1 and $2 \Omega$ ), switching frequency $f_{s w}=$ $330 \mathrm{kHz}$.

Two models of this converter are used in this paper: the averaged nonlinear model and the small-signal linear model. The design of the control laws are based on the small-signal model whereas the averaged model, if not otherwise stated, is used for simulations.

The averaged model was obtained by considering the differential equations of the circuit in Fig. 1.

$$
\left\{\begin{array}{l}
L_{1} i_{L 1}=-(1-u) \cdot\left(v_{C 1}+v_{C 2}\right)+E \\
L_{2} \dot{i}_{L 2}=u \cdot v_{C 1}-(1-u) \cdot v_{C 2} \\
C_{1} v_{\dot{C} 1}=(1-u) \cdot i_{L 1}-u \cdot i_{L 2} \\
C_{2} v_{\dot{C} 2}=(1-u) \cdot\left(i_{L 1}+i_{L 2}\right)-\frac{v_{C 2}}{R},
\end{array}\right.
$$

where $v_{C 1}$ and $v_{C 2}$ denote the voltages over the capacitances $C_{1}$ and $C_{2}, i_{L 1}$ and $i_{L 2}$ the currents through the inductors $L_{1}$ and $L_{2}, E$ the input voltage and $R$ the load resistance. Equation 1 represents the switched model of the SEPIC; in this model the input variable is the switching signal $u$, which can take values 0 or 1 . The averaged model is obtained by replacing the switching function $u$ by its average $\alpha$, called duty cycle [1]. Equation 2 will be referred to as the nonlinear model throughout the paper.

$$
\left\{\begin{array}{l}
L_{1} \dot{i}_{L 1}=-(1-\alpha) \cdot\left(v_{C 1}+v_{C 2}\right)+E \\
L_{2} \dot{i}_{\dot{L} 2}=\alpha \cdot v_{C 1}-(1-\alpha) \cdot v_{C 2} \\
C_{1} \dot{v}_{\dot{C} 1}=(1-\alpha) \cdot i_{L 1}-\alpha \cdot i_{L 2} \\
C_{2} \dot{v}_{C 2}=(1-\alpha) \cdot\left(i_{L 1}+i_{L 2}\right)-\frac{v_{C 2}}{R}
\end{array}\right.
$$

The small-signal model is obtained by considering the variations from the equilibrium as state variables. This model will be referred to as the linear model throughout this paper.
The equilibrium point is obtained by calculating the values of $i_{L 1}, i_{L 2}, v_{C 1}$ and $v_{C 2}$ when their respective derivatives are zero. The subscript $e$ is introduced to denote a value at equilibrium and the $\sim$-notation is introduced to denote a variation from equilibrium. Based on (2), the following values are obtained, where $\alpha_{e}$ is the average duty cycle at equilibrium, obtained from (2) by setting $v_{C 2}$ at its reference value $v_{C 2}^{*}=3.3 \mathrm{~V}$. This gives $\alpha_{e}=0.4231$

$$
\begin{aligned}
i_{L 1 e} & =\left(\frac{\alpha_{e}}{1-\alpha_{e}}\right)^{2} \cdot \frac{E_{e}}{R}, i_{L 2 e}=\frac{\alpha_{e}}{1-\alpha_{e}} \cdot \frac{E_{e}}{R}, \\
v_{C 1 e} & =E_{e}, v_{C 2 e}=\frac{\alpha_{e}}{1-\alpha_{e}} \cdot E_{e} .
\end{aligned}
$$

For all variables in the system the following hold: $\alpha=\alpha_{e}+\widetilde{\alpha}$, $E=E_{e}+\widetilde{E}, R=R_{e}+\widetilde{R}, i_{L 1}=i_{L 1 e}+\widetilde{i_{L 1}}, i_{L 2}=i_{L 2 e}+\widetilde{i_{L 2}}$, $v_{C 1}=v_{C 1 e}+\widetilde{v_{C 1}}$ and $v_{C 2}=v_{C 2 e}+\widetilde{v_{C 2} 2}$.

Here $E_{e}=4.5 \mathrm{~V}$ and $R_{e}=1.3 \Omega$. By use of (2) and (3) and by linearizing the equations, the following system is obtained:

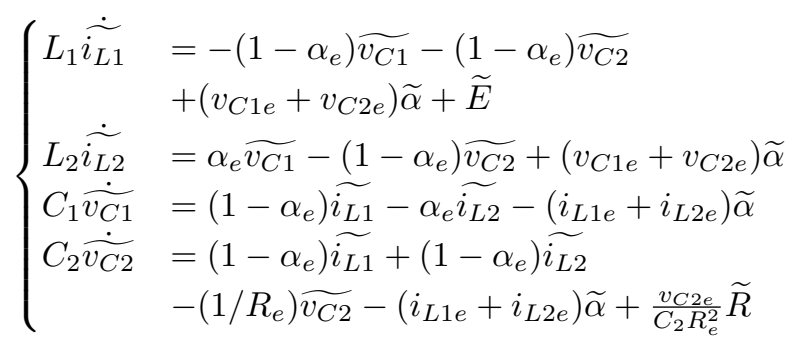

Equation 4 can be written in the following state-space representation:

$$
\dot{\mathbf{x}}=\mathbf{A} \cdot \mathbf{x}+\mathbf{B} \cdot \mathbf{u},
$$

where $\mathbf{x}=\left[\begin{array}{cccc}\widetilde{i_{L 1}} & \widetilde{i_{L 2}} & \widetilde{v_{C 1}} & \widetilde{v_{C 2}}\end{array}\right]^{T}$ is the small-signal state vector and $\mathbf{u}=\left[\begin{array}{ccc}\widetilde{\alpha} & \widetilde{E} & \widetilde{R}\end{array}\right]^{T}$ is the input vector, out of which $\tilde{\alpha}$ is the control input and parameter variations, $\tilde{E}$ and $\tilde{R}$ are here modelled as disturbances. The matrices $\mathbf{A}$ are $\mathbf{B}$ are given in the Appendix.

\section{STATE-FEEDBACK CONTROL DESIGN}

Here a state-feedback control with integral action able to improve tracking properties and disturbance rejection is considered. This leads to the following state being added to the state-space representation: $\dot{z}=v_{C 2}^{*}-v_{C 2}=v_{C 2}^{*}-C x$. The state-space representation of the extended system can then be written as:

$$
\begin{aligned}
\left(\begin{array}{l}
\dot{x} \\
\dot{z}
\end{array}\right) & =\left[\begin{array}{cc}
A & 0 \\
-C & 0
\end{array}\right]\left(\begin{array}{l}
x \\
z
\end{array}\right)+\left[\begin{array}{c}
B \\
0
\end{array}\right] \cdot u+\left[\begin{array}{l}
0 \\
1
\end{array}\right] \cdot v_{C 2}^{*} \\
y & =\left[\begin{array}{ll}
C & 0
\end{array}\right] \cdot\left(\begin{array}{l}
x \\
z
\end{array}\right)
\end{aligned}
$$

The controller was designed by imposing two of the closedloop poles as a double pole $(\tau s+1)^{2}$ such that to ensure a desired closed-loop settling time (relation $t_{r}=4.75 \tau$ has been used). The three remaining poles were chosen to be 
eight times faster, thereby allowing the system to be primarily determined by the double-pole polynomial. The desired settling time was here chosen to be five times faster than the slowest dynamics of the open-loop system, which gives an imposed settling time of $0.31 \mathrm{~ms}$. This gives the feedback gain $L=\left[\begin{array}{lllll}0.4976 & -0.2166 & 0.1776 & 0.1694 & -4.0669\end{array}\right]$.

\section{IV. $H_{\infty}$ CONTROL DESIGN}

\section{A. Specifications}

$H_{\infty}$ control method consists of shaping the desired characteristics of the closed-loop system by using suitably shaped templates for the desired closed-loop frequency responses. Templates are specified by means of weighting functions on the various input-output channels.

The sensitivity functions are defined as follows:

$$
\begin{aligned}
S & =\frac{I}{I+G K}, \quad T=I-S, \quad K S=K \cdot S, \\
S_{E} & =G_{E} \cdot S, \quad S_{R}=G_{R} \cdot S,
\end{aligned}
$$

where $G, G_{E}$ and $G_{R}$ are the transfer functions from the control input $\widetilde{\alpha}$ and disturbances $\widetilde{E}$ and $\widetilde{R}$ to the output $\widetilde{v_{C 2}}$. The transfer function $K$ is the designed controller.

As regards the template imposed for function $S$, the rise time of the closed-loop system can be estimated by [2]

$$
t_{r} \approx \frac{2.3}{\omega_{T}},
$$

where $\omega_{T}$ is the bandwidth of the complementary sensitivity function $T$, which is defined as the frequency where $\|T(j \omega)\|$ crosses $-3 \mathrm{~dB}$ from above [2]. Under the assumption that the closed-loop system has no oscillations, the approximation $\omega_{S}=\omega_{T}$ is made, where $\omega_{S}$ is the bandwidth of the sensitivity function and is defined as the frequency where $\|S(j \omega)\|$ crosses $-3 \mathrm{~dB}$ from below. The rise time is assumed to be approximately equal to the settling time. The desired closedloop settling time is here chosen to be ten times faster than that of the open-loop system, which gives a desired settling time of $0.155 \mathrm{~ms}$.

$M_{S}$ is the maximum peak of $S\left(\|S\|_{\infty}\right)$ and is related to the robustness margin of the system. A choice of $M_{S}=2$ in the weighting function for $S$ guarantees a gain margin $G M \geq 2$ and phase margin $P M \geq 29.0^{\circ}$ [2].

The weighting function for $S$ is chosen as

$$
W_{e}(s)=\frac{s / M_{S}+\omega_{S}}{s+\omega_{S} \epsilon},
$$

where the parameter $\epsilon$ is chosen as $1 / 100$ to limit the maximum steady-state error. The weighting function for $K S$ is chosen in a similar manner by specifying a maximum gain $M_{u}=1$ (to limit actuator saturation), a roll-off frequency $\omega_{b c}=400 \mathrm{rad} / \mathrm{s}$ (to start attenuating noise with a frequency higher than $400 \mathrm{rad} / \mathrm{s}$ ) and a maximum amplification of noise $\epsilon_{1}=0.5$. The weighting function for $K S$ is:

$$
W_{u}(s)=\frac{s+\omega_{b c} / M_{u}}{\epsilon_{1} s+\omega_{b c} \epsilon}
$$

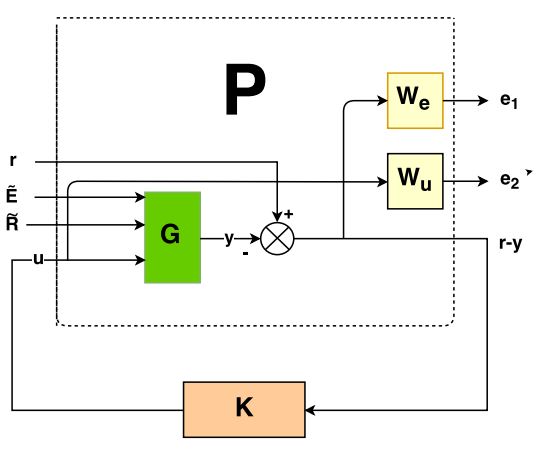

Figure 2. The generalized plant $P$ and the controller $K$ in the P-K form

Simulations of the closed-loop system have shown highfrequency oscillations in the control input $\alpha$, which could prove unfeasible, even with high-frequency PWM signals. Thus, a second $H_{\infty}$ controller is designed with the following modified specifications for $S: \epsilon=1 / 1000$ to limit the steadystate error to $1 / 1000$, while $\omega_{S}$ and $M_{S}$ remained unchanged. The specifications for $K S$ were chosen as: $M_{u}=0.0738$ (to limit the maximum peak of the control input in response to a reference variation), $\epsilon_{1}=0.01$ (to limit the amplification of noise in high frequencies) and $\omega_{b c}=300 \mathrm{rad} / \mathrm{s}$ (the frequency where $1 / W_{u}$ crosses $0 \mathrm{~dB}$ from above, chosen to attenuate high-frequency noise).

\section{B. Controller Design}

Recall that the control goal is to regulate the output voltage at a reference value, denoted here as $v_{C 2}^{*}$, in spite of parameter variations, modelled as disturbances.

The definition of the control problem to be solved in the $H_{\infty}$ framework is shown in Fig 2. Here $r=v_{C 2}^{*}$ is the reference input, $y=\widetilde{v_{C 2}}$ the measured output and $e_{1}$ and $e_{2}$ the controlled outputs. The internal structure of the generalized plant is shown within the dashed lines. The $H_{\infty}$ control problem to be solved is to find a controller $K$ such that

$$
\left\|\begin{array}{ccc}
W_{e} S & -W_{e} G_{E} S & -W_{e} G_{R} S \\
W_{u} K S & -W_{u} K G_{E} S & -W_{u} K G_{R} S
\end{array}\right\|_{\infty} \leq \gamma,
$$

where $\gamma \leq 1$ means that the templates, as specified by the weighting functions, are satisfied. The transfer functions $G$, $G_{E}$ and $G_{R}$ were defined in the beginning of this section. The design of the generalized plant and the $H_{\infty}$ synthesis were carried out in MATLAB ${ }^{\circledR}$ Robust Control Toolbox. The value of $\gamma$ obtained for the first controller is 0.8920 , meaning the templates are satisfied with some margin.

However, the specifications for the second controller were not met and a value of 21.6495 was obtained for $\gamma$. This controller will be called controller 2 throughout the remainder of this paper, whereas the other controller will be referred to as controller 1. 
a) Output voltage $\left(\widetilde{v_{C 2}}\right)$

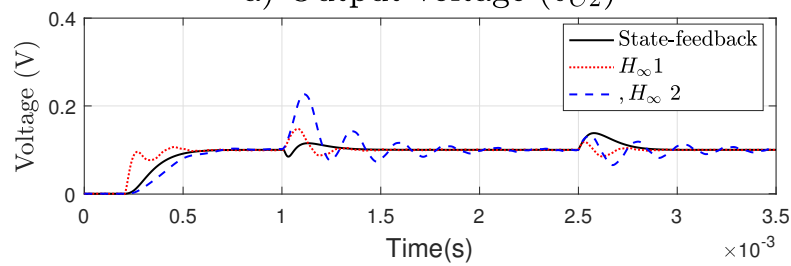

b) Control input $\alpha$ - State-feedback

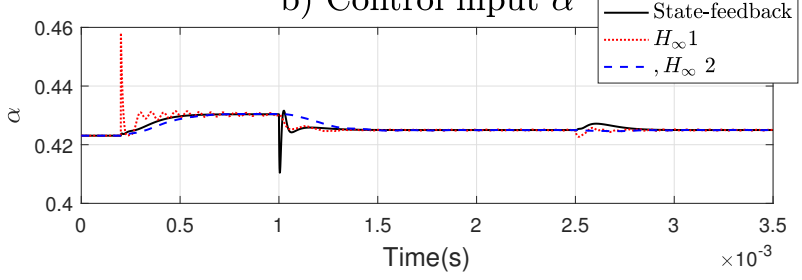

Figure 3. Comparison for the case of reference step input of 0.1 at $0.2 \mathrm{~ms}$, a step input of $0.1 \mathrm{~V}$ in $\widetilde{E}$ at $1 \mathrm{~ms}$ and a step input of $0.1 \Omega$ of $\widetilde{R}$ at $2.5 \mathrm{~ms}$. a) Output voltage $\left(\widetilde{v_{C 2}}\right)$ b) Control input $(\alpha)$

\section{SIMULATION RESULTS}

This section is dedicated to a comparative numericalsimulation analysis of the two robust controllers against the integral state-feedback control.

Fig. 3 shows the step response and disturbance rejections of the state-feedback controller and the two $H_{\infty}$ controllers. The first $H_{\infty}$ controller has the fastest rise time, whereas the state-feedback controller and the second $H_{\infty}$ controller are the slowest. The first $H_{\infty}$ controller has the best disturbance rejection, followed by the state-feedback controller and the second $H_{\infty}$ controller. However, this very good performance is achieved with quite abrupt variations of the average duty cycle, which is to avoid, even if magnitudes remain within limits (here, between 0 and 1 ).

On the other hand, the second $H_{\infty}$ controller has the smoothest control input.

\section{SENSITIVITY AND ROBUSTNESS ANALYSIS}

In order to analyze the performance of the controllers, the sensitivity functions defined in Section IV were used. The tools provided by $\mu$-analysis were used to evaluate robust stability and performance.

\section{A. State-Feedback Controller}

Fig. 4 and Fig. 5 show the sensitivity functions for the state-feedback controller. The peak of $\|S\|$ is below 2, which implies some robust stability margin. The unitary gain at $\|T(0)\|$ indicates good tracking properties. The rejection of disturbances with no steady-state error is seen in $S_{E}$ and $S_{R}$. The structured singular value, denoted $\mu$, is used to analyze the stability of the closed-loop system within the uncertainty ranges of $R$ and $E$. A value of $\mu<1$ means that the closedloop system remains stable within the uncertainty range. Fig. 6 shows that the system is robustly stable. Simulations have shown that this also holds true for the nonlinear system. a) $S$

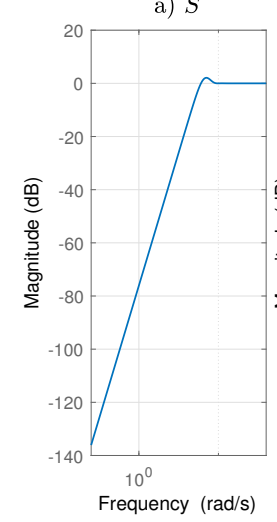

b) $T$

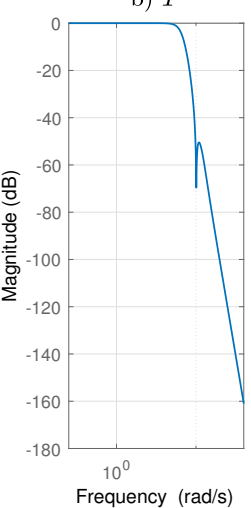

c) $K S$

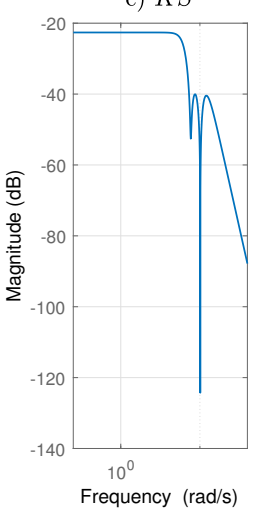

Figure 4. Sensitivity functions for the closed-loop system with state-feedback controller a) Sensitivity function $S$ b) Complementary Sensitivity function $T$ c) Sensitivity function $K S$
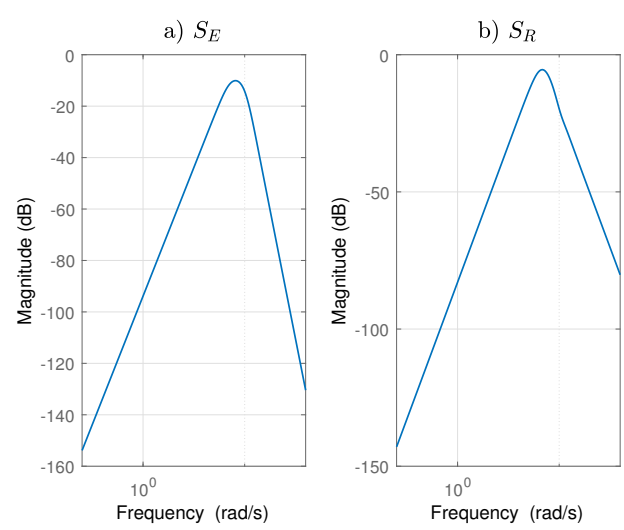

Figure 5. Sensitivity functions for the closed-loop system with state-feedback controller a) Sensitivity functions $S_{E}$ from disturbance input $\tilde{E}$ to output $y$ b) a) Sensitivity functions $S_{R}$ from disturbance input $\tilde{R}$ to output $y$

The performance of the state-feedback controller was also evaluated by varying the values of the input voltage $E$ and load resistance $R$. The results showed that the desired performance, namely the settling time, is not guaranteed to remain the same within the specified uncertainty range. Thus, one can say that the state-feedback integral control does not ensure robust performance.

\section{B. $H_{\infty}$ Controller}

The sensitivity functions were used to analyze the characteristics of the system. Fig. 7 and 8 show the sensitivity functions for the two $H_{\infty}$ controllers together with the templates for controller 2. The decreased gain of $K S$ at high frequencies implies an attenuation of high-frequency oscillations in the control input. However, the lower bandwidth of the second controller indicates a slower rise time. The second controller's amplification of disturbances can be seen as a gain above $0 \mathrm{~dB}$ in $S_{E}$ and $S_{R}$.

The second $H_{\infty}$ controller is a better candidate for a possible practical implementation as its control input presents some 


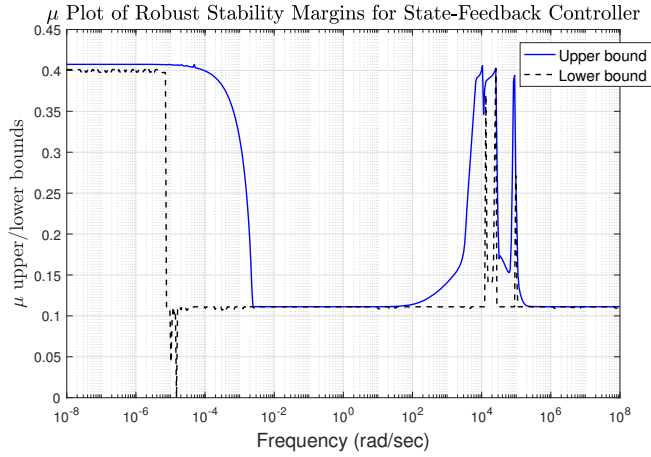

Figure 6. Upper and lower bounds of $\mu$ for the closed-loop system with state-feedback controller a) $S$

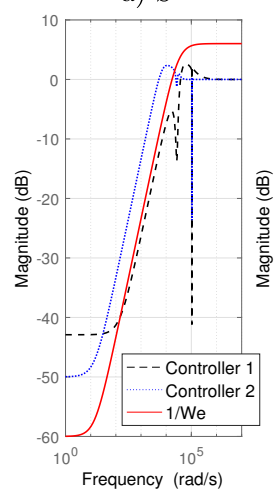

b) $T$

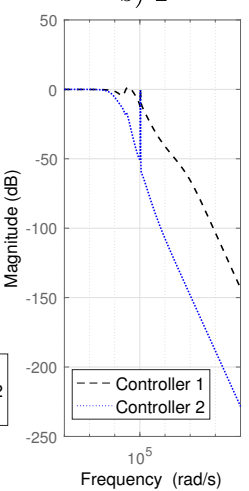

c) $K S$

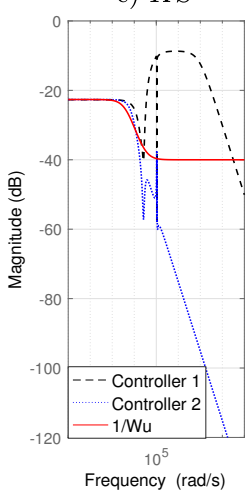

Figure 7. Sensitivity functions for the closed-loop system with the two $H_{\infty}$ controllers a) Sensitivity function $S$ b) Complementary Sensitivity function $T$ c) Sensitivity function $K S$

smoother variations, more likely to be achieved in practice. Thus, the robust stability and performance of the closed-loop system with this second $H_{\infty}$ controller were evaluated. The results of the $\mu$-analysis for stability and performance are presented in Fig. 9. These results suggest that the controller is robustly stable. However, this is only valid for the linear system. Simulations performed with the nonlinear system have shown that the closed-loop system is not stable for all values within the specified uncertainty range. Based on the simulation results, it was found that the nonlinear closed-loop system is stable for values $E \in[3.3,5.7] \mathrm{V}, R \in[1,2] \Omega$. Fig. 10 shows that the parameter domain of variation is split into two regions from both robust-stability and robust-performance viewpoints.

The results of the $\mu$-analysis for robust performance show that the desired performance, as specified by the controller's $\gamma$-value, is not obtained for the whole uncertainty range. Fig. 10b) allows to identify the region where the gain of the closed-loop system remains below the value of $\gamma$, that is, the closed-loop system is robust in performance. Fig. 11 shows simulation results illustrating the case of an unstable closedloop system, which confirms results of analysis. a) $S_{E}$

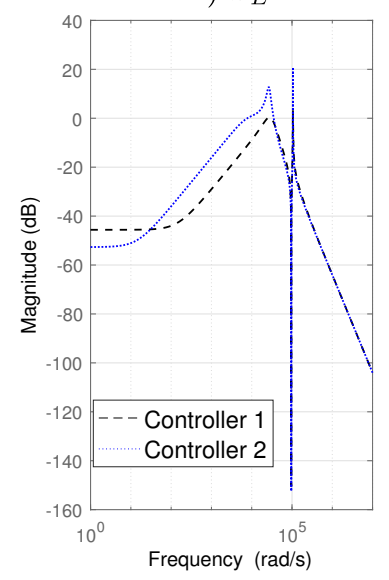

b) $S_{R}$

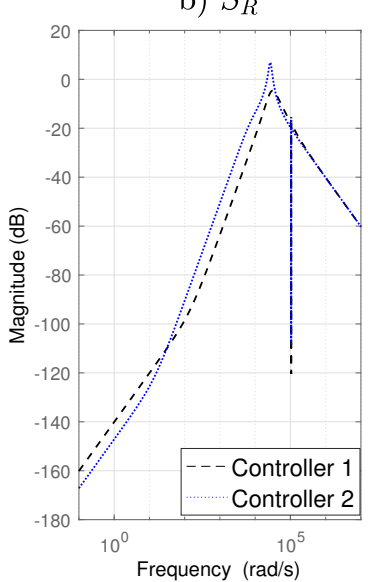

Figure 8. Sensitivity functions for the closed-loop system with the two $H_{\infty}$ controllers a) Sensitivity functions $S_{E}$ from disturbance input $\tilde{E}$ to output $y$ b) Sensitivity functions $S_{R}$ from disturbance input $\tilde{R}$ to output $y$ a) $\mu$ Stability Margins

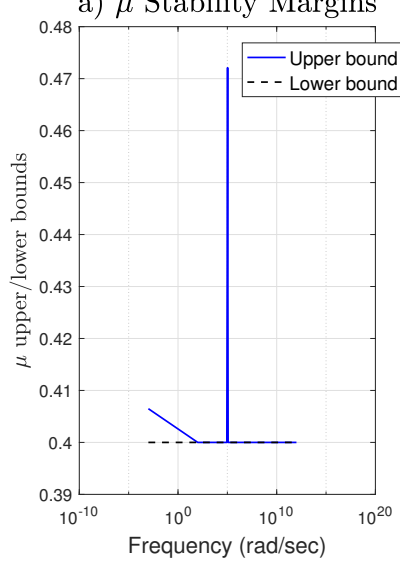

b) $\mu$ Performance Margins

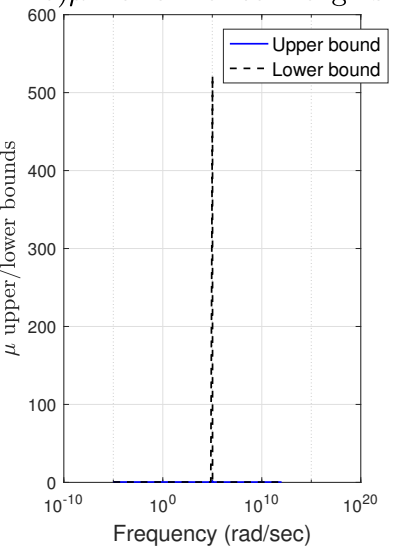

Figure 9. Upper and lower bounds of the $\mu$ value for the second $H_{\infty}$ controller a) Robust stability b) Robust performance

\section{CONCLUSIONS}

This paper has approached the topic of robust control for DC-DC power converters. $H_{\infty}$ formalism has been chosen as framework for stating the output voltage regulation problem in presence of parameter uncertainties as a disturbance-rejection problem; the single-ended primary-inductor converter (SEPIC) has been presented as a case study. Design, analysis and numerical simulations in MATLAB ${ }^{\circledR} /$ Robust Control Toolbox have allowed assessment of this approach against a classical integral state-feedback control from the point of view of robustness in stability and in performance, when two parameters - input voltage and load resistance - vary, but are bounded within known ranges. Both these approaches consider the linearized model around a certain, desired, steady-state point; but in simulation the nonlinear averaged model has been used for both. Good results concerning robust stability have been obtained for the classical control; however, robust performance has not been guaranteed with this control for the whole param- 

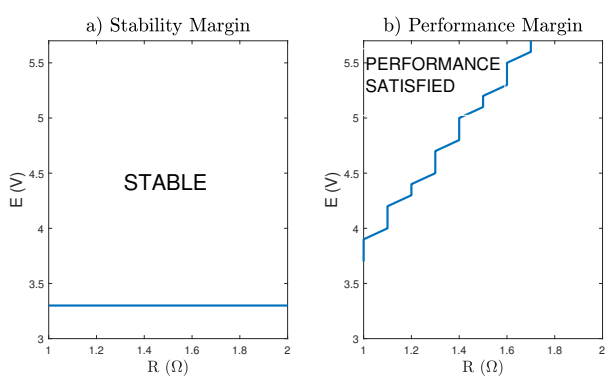

Figure 10. Regions of stability (a) and performance (b) for the second $H_{\infty}$ controller

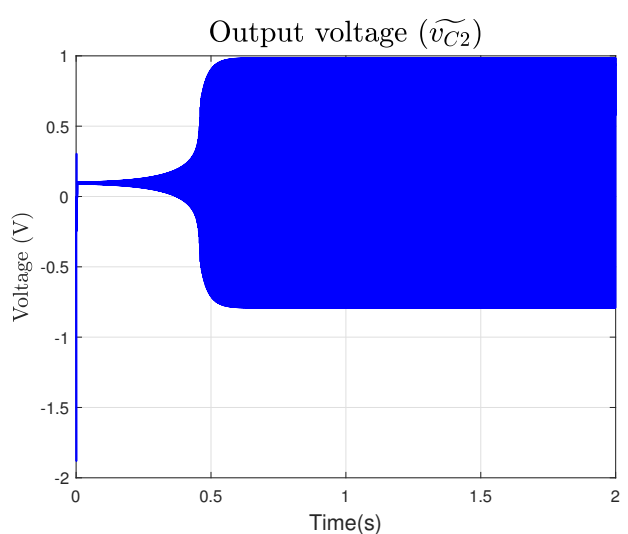

Figure 11. Simulation of the closed-loop system with the second $H_{\infty}$ controller for the unstable case $E=3 \mathrm{~V}$ and $R=1.3 \Omega$

eter variation domain. Robust control formalism is able to offer more detailed information with this respect. Thus, by using the $\mu$-analysis - followed by confirmation by numerical simulation - one can identify precisely the parameter domain within which the closed-loop system remains stable and keeps the same performance quality. Indeed, $H_{\infty}$ provides more degrees of freedom, but this advantage has to be weighed against its complexity compared to state-feedback control. Depending on application complexity, requirements and constraints one or the other might be the preferred choice.

\section{APPENDIX A}

Matrices $\mathbf{A}$ and $\mathbf{B}$ of the small-signal model.

$$
\begin{aligned}
& \mathbf{A}=\left[\begin{array}{cc}
0 & 0 \\
0 & 0 \\
\left(1-\alpha_{e}\right) / C_{1} & -\alpha_{e} / C_{1} \\
\left(1-\alpha_{e}\right) / C_{2} & \left(1-\alpha_{e}\right) / C_{2}
\end{array}\right. \\
& -\left(1-\alpha_{e}\right) / L_{1} \quad-\left(1-\alpha_{e}\right) / L_{1} \\
& \alpha_{e} / L_{2} \quad-\left(1-\alpha_{e}\right) / L_{2} \\
& 0 \\
& 0 \\
& -1 /\left(R C_{2}\right) \\
& \mathbf{B}=\left[\begin{array}{ccc}
\left(v_{C 1 e}+v_{C 2 e}\right) / L_{1} & 1 / L_{1} & 0 \\
\left(v_{C 1 e}+v_{C 2 e}\right) / L_{2} & 0 & 0 \\
-\left(i_{L 1 e}+i_{L 2 e}\right) / C_{1} & 0 & 0 \\
-\left(i_{L 1 e}+i_{L 2 e}\right) / C_{2} & 0 & v_{C 2 e} /\left(C_{2} R_{e}^{2}\right)
\end{array}\right]
\end{aligned}
$$

\section{REFERENCES}

[1] S. Bacha, I. Munteanu, and A. I. Bratcu, Power electronic converters modeling and control - with case studies. London ; Heidelberg ; New York : Springer, 2013.

[2] S. Skogestad and I. Postlethwaite, Multivariable feedback control : Analysis and design. Hoboken, NJ : John Wiley, cop., 2005.

[3] T. Glad and L. Ljung, Reglerteknik : Grundläggande teori. Lund : Studentlitteratur, 2006.

[4] J. Lam and P.K.Jain, "A high-power-factor single-stage single-switch electronic ballast for compact fluorescent lamps", IEEE Transactions on Power Electronics, vol. 25, no. 8, pp. 2045-2058, 2010.

[5] B. Geethalakshmi, "Design of a closed loop control scheme for a dc-dc sepic converter using loop shaping method", in 2015 International Conference on Circuits, Power and Computing Technologies [ICCPCT-2015], 2015, pp. 1-5.

[6] P. Omer, J. Kumar, and B. S. Surjan, "Design of robust pid controller for buck converter using bat algorithm", in 2016 IEEE 1st International Conference on Power Electronics, Intelligent Control and Energy Systems (ICPEICES), 2016, pp. 1-5.

[7] M. Fard and M. Aldeen, "Robust control design for a boost converter in a photovoltaic system", in 2016 IEEE 7th International Symposium on Power Electronics for Distributed Generation Systems (PEDG), 2016, pp. 1-9.

[8] M. Salimi and V. Hajbani, "Robust closed loop control of the transformerless dc-dc converters with high step up voltage gain", in 2016 IEEE PES Innovative Smart Grid Technologies Conference Europe (ISGT-Europe), 2016, pp. 1-5.

[9] R. Ling, D. Maksimovic, and R. Leyva, "Second-order sliding-mode controlled synchronous buck dc-dc converter", IEEE Transactions on Power Electronics, vol. 31, no. 3, pp. 2539-2549, 2016.

[10] S. Zhuo and Y. Huangfu, "Robust buck converter design with a high-order sliding mode differentiator", in IECON 2015 - 41st Annual Conference of the IEEE Industrial Electronics Society, 2015, pp. 000 162-000 167. 